\title{
Multiparameter Stochastic Dynamics of Ecological Tourism System with Continuous Visitor Education Interventions
}

\author{
Dongping Wei, ${ }^{1}$ Qunming Zheng, ${ }^{2}$ and Shouwen Wen ${ }^{3}$ \\ ${ }^{1}$ Math and Physics Department, Shenzhen Polytechnic, Shenzhen 518055, China \\ ${ }^{2}$ College of Tourism, Hunan Normal University, Changsha 410081, China \\ ${ }^{3}$ School of Management, Shenzhen Polytechnic, Shenzhen 518055, China
}

Correspondence should be addressed to Qunming Zheng; zqmwalter@163.com

Received 6 February 2015; Accepted 16 April 2015

Academic Editor: Sergio De Rosa

Copyright (C) 2015 Dongping Wei et al. This is an open access article distributed under the Creative Commons Attribution License, which permits unrestricted use, distribution, and reproduction in any medium, provided the original work is properly cited.

\begin{abstract}
Management of ecological tourism in protected areas faces many challenges, with visitation-related resource degradations and cultural impacts being two of them. To address those issues, several strategies including regulations, site managements, and visitor education programs have been commonly used in China and other countries. This paper presents a multiparameter stochastic differential equation model of an Ecological Tourism System to study how the populations of stakeholders vary in a finite time. The solution of Ordinary Differential Equation of Ecological Tourism System reveals that the system collapses when there is a lack of visitor educational intervention. Hence, the Stochastic Dynamic of Ecological Tourism System is introduced to suppress the explosion of the system. But the simulation results of the Stochastic Dynamic of Ecological Tourism System show that the system is still unstable and chaos in some small time interval. The Multiparameters Stochastic Dynamics of Ecological Tourism System is proposed to improve the performance in this paper. The Multiparameters Stochastic Dynamics of Ecological Tourism System not only suppresses the explosion of the system in a finite time, but also keeps the populations of stakeholders in an acceptable level. In conclusion, the Ecological Tourism System develops steadily and sustainably when land managers employ effective visitor education intervention programs to deal with recreation impacts.
\end{abstract}

\section{Introduction}

According to the 2014 Yearbook of China Tourism Statistics, China enjoys the largest domestic tourism market in the world. Its inbound tourism market ranks the fourth and its outbound market ranks the first in the Asian-Pacific region. A large population gathers on tourism destinations in public vocational days including the Golden Weeks during the Labor Day (May 1) and National Day (October 1). Visitors crowd into the protected areas such as Huangshan National Natural Protected Park, Zhangjiajie National Forest Park, and Tai Mountain. These parks are so populated that it is hard to even walk. Due to the lack of visitor educational interventions, the parks suffer from quick unrecoverable degradation of resources. For example, litters are observed everywhere: precious trees are damaged, and wild animals are badly disturbed. The social impacts are no less: tensions among tourists due to crowding in the parks, conflicts between visitors and park managers, and, in an extreme case, tens of thousands of tourists trapped in Huashan Mountain, Shaanxi province, on October 2, 2012. In this case, a huge number of visitors challenged the capacity of the cable cars at Huashan Mountain, leaving tens of thousands stuck at the mountain peak late on Tuesday night (see http://english.cri.cn/6909/ 2012/10/04/2821s725417.htm). According to China Central Television, restless visitors demanded refunds from the tourism committee, and police was dispatched to help dealing with the crisis. Clearly, the negative resource and social impacts diminish the visitors' satisfaction quickly.

Hence, the fast expanding visitation in some protected areas severely challenges the protection of the environment and precious natural resources, cultural resources, and social conditions. The United State faced the same situation in the 1970s. To address this issue, the land managers of protected areas, tourism providers, and other stakeholders commonly employed regulations and site management. As a direct 
management measure, the regulations curtailed visitors' freedom and emphasized on the potential enforcement with punitive actions [1]. However, the enforcement was reported to be costly and lacks efficacy and antagonize visitors [2]. Site managements including developing and hardening a recreation site or trail are less direct, but they were also costly and could permanently alter the natural landscape and the nature of recreation experiences $[3,4]$. In contrast, a visitor education program is designed to persuade visitors to adopt low impact practices. It is considered as a more appropriate, light-handed, and indirect management response to reduce resource impacts and improve visitor experiences [5]. Many visitor education programs such as Leave No Trace [6-8], Code of Conduct [9], and Environmental Guidelines for Tourists [10] have already been applied in many countries. But it is a pity that there has been no such programs being mandated in china where the tourism market is extremely booming and the resource and social impacts are serious. The advantages of visitor education programs have been recognized by more and more Chinese scholars and site managers in recent years. Some pilot studies of the visitor educational intervention to protect precious natural and cultural resource have been reported [11-13].

Many scholars from the U.S., Australia, and Canada have discussed the education messages contents (see, e.g., [1416]), deliveries (see, e.g., [17-21]), audience characteristics (see, e.g., [22-24]) and theoretical grounding (see, e.g., [2527]), the efficacy of educational efforts (see, e.g., $[10,28]$ ), and so on. However, most of the studies only focus on the empirical results obtained by visitor education interventional experiment data [6]. Few researches were able to quantify the efficacy of the visitor education intervention in tourism management. The purpose of this investigation is to study the dynamics of Ecological Tourism System and the effects of visitor education programs on the population dynamics by using a stochastic dynamics model.

An Ecological Tourism System consists of the natural resources including air, rocks, mountains, soils, and trees and the stakeholders in visitation destination. The stakeholders [11] in ecotourism include the following seven groups:

(1) the tourism resource supply group, including tourism resource managers and park managers;

(2) the visitation product sellers group, including tourism companies and tourism website managers;

(3) the group of related products or services suppliers, including sellers who are providing transportations, hotels, catering, and other tourism memories products;

(4) the group of local communities, including the staff of tourism companies and workers in other companies and local people;

(5) the government group, including center government, provincial governments, and municipalities;

(6) the special interest group, which are often shown as nongovernment organizations (NGOs) including environmental organizations, human rights and labor rights organizations, Trust and environmental charity institutes, academics, and social media;

(7) visitors, which are the core consumers of tourism products and services.

The stakeholders in ecotourism destinations cannot survive without the support from one another, and they must unite to a population in a certain space [11]. It is valuable to study the dynamic behavior of the Ecological Tourism System under the visitor educational intervention. Wei et al. (2013) proposed a stochastic dynamics to study the efficacy of visitor education interventions [29]. Applying stochastic differential equation theory [30], they proved that the Ecological Tourism System can develop sustainably when perturbed by the continual visitor educational intervention. But they only considered one parameter of the system that was perturbed by visitor education intervention. This study follows the same thread of research in Wei et al. (2013) and further assumes that three parameters (i.e., the intrinsic increasing rate of stakeholders, the immigration rate of stakeholders from outside of the system, and the emigration rate of stakeholders from the inner system) are perturbed by the visitor education intervention stochastically. The Multiparameter Stochastic Dynamics of Ecological Tourism System (MPSDETS) is proposed to study the efficacy of the visitor educational programs.

In order to clearly present Multiparameters Stochastic Dynamics of Ecological Tourism System in the following three sections, the key notations and definitions of variables are introduced as follows:

$x(t)$ : stakeholders in Ecological Tourism System at time $t$;

$x_{0}$ : initial value of Ecological Tourism System at time $t_{0}$

$\alpha$ : intrinsic increasing rate of stakeholders within Ecological Tourism System;

$\beta$ : immigration rate of stakeholders from outside of Ecological Tourism System;

$\gamma$ : emigration rate of stakeholders from the inner Ecological Tourism System;

$K$ : maximum carrying capacity of Ecological Tourism System;

$\omega_{i}(i=1, \ldots, 4)$ : Brownian motion;

$\varepsilon_{i}(i=1, \ldots, 4)$ : intensity of visitor education interventions;

$\tau_{e}$ : explosion time of stochastic differential equation;

$\tau_{k}$ : stopping time of stochastic differential equation;

$k_{0}$ : a sufficient large number;

$k$ : any nonnegative integer;

$V(x)$ : a $C^{2}$-function $V: R^{+} \rightarrow R^{+}$;

$M$ : a sufficient large positive number;

$T$ : a sufficient large positive constant. 
We define the system stability state, collapsed state, and chaos state as follows. When the number of stakeholders $x(t)$ is smaller than the maximum capacity of system, then the system is under stability sate. When $x(t)$ explodes to infinity or $x(t)<0$, then the system collapsed. When $x(t)$ is a little larger than or near the maximum capacity of system, then the system is under chaos state. When the system is under stability state, then there are proper stakeholders in the system and the ETS are running safely. When the system is under chaos state, then there are too much stakeholders in the system and the ETS are running dangerously. When the system is under collapsed state, then the system cannot accept any visitors and the ETS are damaged seriously.

\section{The Multiparameter Stochastic Dynamics of Ecological Tourism System}

There are $x(t)$ stakeholders in the Ecological Tourism System (ETS) at time $t$. Some stakeholders may enter the system from outside of ETS while some others leave the system after they finished their visitation activities or business affairs. $K$ is the maximum carrying capacity of the ETS, and then $1 / K$ is the recreation impacts factor per capita. Then, $x(t) / K$ means the recreation impact factor at time $t$. The Ordinary Differential Equation of Ecological Tourism System (ODETS) without visitor educational intervention can be expressed as

$$
\frac{d x}{d t}=x\left(\alpha+\beta-\gamma-\frac{1}{K} x\right) .
$$

The solution of Ordinary Differential Equation (1) cannot avoid explosion when given a large initial value $x_{0}$.

Since the variable $x(t)$ denotes the population of stakeholders, $x(t)$ must be positive value on time $t \geq 0$. When the parameters of (1) $\alpha, \beta, \gamma \in(0,1), K \in R^{+}$, (1) only has a local solution as follows:

$$
\begin{aligned}
& x(t) \\
& =\frac{\alpha+\beta-\gamma}{-(1 / K)+e^{-(\alpha+\beta-\gamma) t}\left(\alpha+\beta-\gamma-(1 / K) x_{0}\right) / x_{0}} .
\end{aligned}
$$

Given that $x_{0}>0$, it is not difficult to see that $x(t)$ explodes to infinity when $t \rightarrow T$

$$
T=-\frac{1}{\alpha+\beta-\gamma} \log \frac{(1 / K) x_{0}}{\left(\alpha+\beta-\gamma-(1 / K) x_{0}\right)} .
$$

Hence, the solution of (1) $x(t)$ explodes to infinity in a finite time $T$. The initial quantity of visitors $x_{0}$ is very large in some protected area especially on the public vocation in China as mentioned in Section 1. So, the solution of the differential dynamic equation explodes inevitably. In other words, the ETS is unstable and easy to collapse. The Ecological Tourism System cannot develop sustainably in that case.

Now, consider the case that ETS is intervened by visitor education. In this case, the intrinsic rate of increase is stochastically perturbed with

$$
\alpha \longrightarrow \alpha+\varepsilon \dot{\omega}_{t},
$$

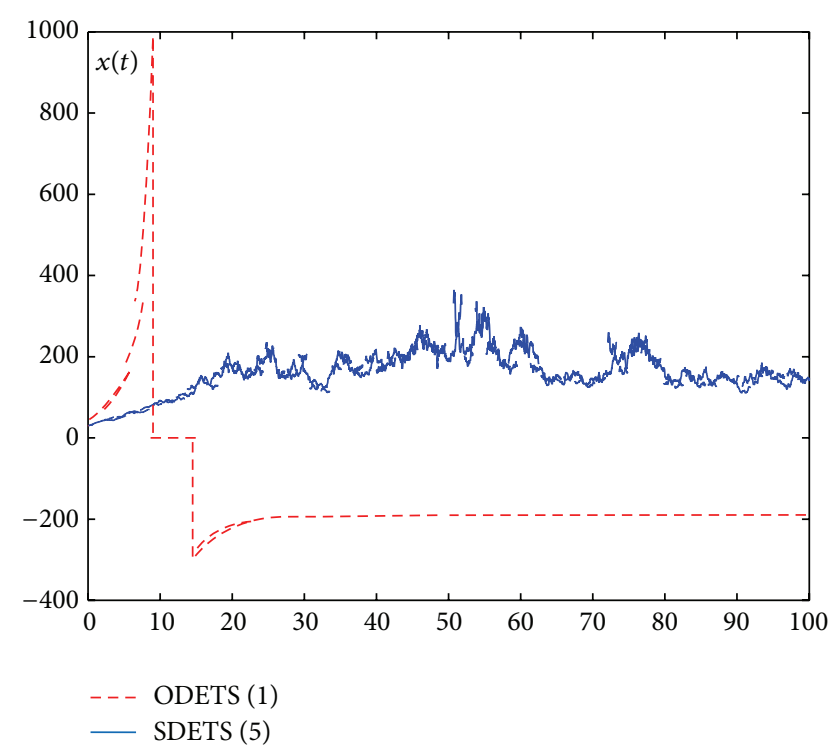

FIGURE 1: The simulations of ODETS (1) and SDETS (5) when $\alpha=$ $0.15, \beta=0.08, \gamma=0.06, K=1000$, and $x_{0}=30$.

where $\dot{\omega}_{t}=d \omega(t)$. As a result, the stochastic dynamic model of Ecological Tourism System (SDETS) with one stochastic parameter $\alpha \rightarrow \alpha+\varepsilon \dot{\omega_{t}}$ can effectively suppress the explosion of the solution of (1) in a finite time with any initial value $x_{0}$

$$
d x=x\left(\alpha+\beta-\gamma-\frac{1}{K} x\right) d t+\varepsilon x^{2} d \omega(t) .
$$

Figure 1 shows that ODETS (1) without visitor educational intervention explodes at time $t=9.53$ when $\alpha=0.15$, $\beta=0.08, \gamma=0.06, K=1000$, and $x_{0}=30$. However, SDETS (5) does not explode at any time with the same parameters shown in Figure 1. Similar results can be drawn from Figure 2 for the case with parameters $\alpha=0.25, \beta=0.09, \gamma=$ $0.06, K=1000$, and $x_{0}=40$. ODETS (1) explodes at time $t=5.64$ as shown in Figure 2, whereas the stochastic system is more tempered. However, Figure 2 also shows that the number of population of the stochastic system reaches a large number (1083), greater than the maximum capacity carrying of the system at $t=5.64$. Although the value of stakeholders $x(t)$ returns shortly to a normal level in a short time, the system is unstable or in chaos at the small interval time $t \in[147,149]$. It is obvious to see that SDETS with single-parameter intervention is not effective to describe the system at some time. Hence, it is valuable to improve SDETS (5) to avoid the chaos situation.

It is easy to find that other parameters $\beta, \gamma, K$ in (1) can also affect the stability of the ETS. And these parameters are also stochastically perturbed by visitor educational intervention. It is important to study how the stochastic parameters affect Ecological Tourism System.

Because many stakeholders from outside competitors can immigrate into the system randomly, the immigration rate of the system may be larger than the acceptable level without visitor education intervention. If the immigration rate of the system $\beta$ is too large, then the stakeholders increase too 


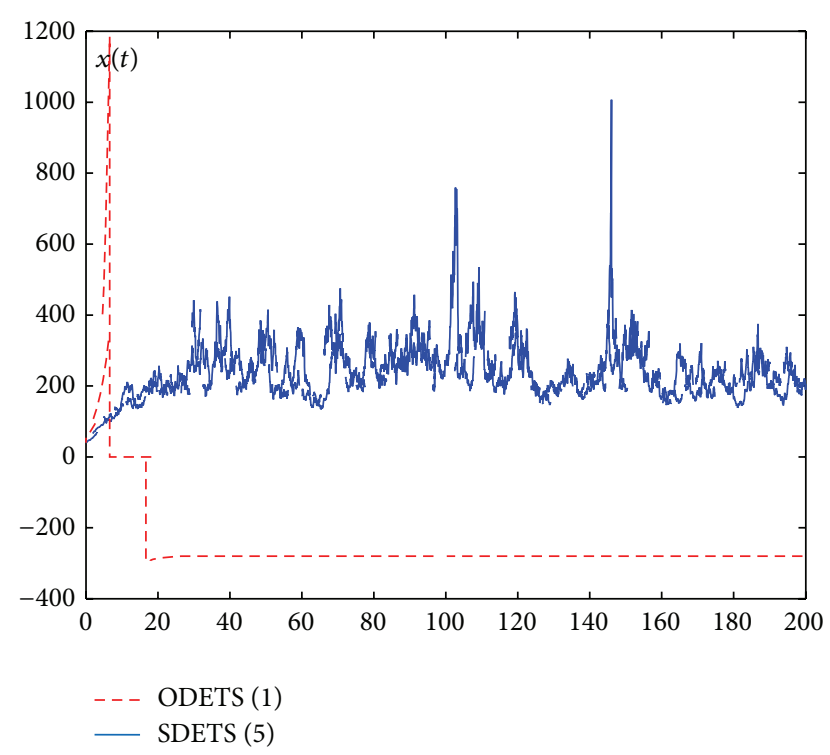

FIGURE 2: The simulations of ODETS (1) and SDETS (5) when $\alpha=$ $0.25, \beta=0.08, \gamma=0.06, K=1000$, and $x_{0}=40$.

fast to over the limit capacity in a short time leading to the chaos situation of the system. In fact, the immigration rate can be suppressed by the visitor education intervention. The effective visitor education programs can induce visitors and other stakeholders entering the system orderly, but the effects are changing stochastically. So, the immigration rate $\beta$ is perturbed by visitor education intervention stochastically as follows:

$$
\beta \longrightarrow \beta+\varepsilon_{2} \omega_{2}(t)
$$

where $\omega_{2}(t)$ is a Brownian motion and $\varepsilon_{2}$ is the intensity of visitor education.

Stakeholders such as visitors and other relative product suppliers can leave ETS when they have finished their activities and business. The emigration rate of stakeholders $\gamma$ may be lower than the acceptable level of the system without visitor educational intervention. Then, the system may be too crowded to keep developing sustainably. The immigration rate of stakeholders $\gamma$ can be restored to normal level because the effective visitor education programs can lead visitors and other stakeholders to leave ETS in time. But the effects of visitor education are changing stochastically in different situation. So, the immigration rate of stakeholders $\gamma$ also fluctuates according to the effects of visitor education interventions

$$
\gamma \longrightarrow \gamma+\varepsilon_{3} \omega_{3}(t)
$$

where $\omega_{3}(t)$ is a Brownian motion and $\varepsilon_{3}$ is the intensity of visitor education to emigration rate of stakeholders.

The visitors and other stakeholders within ETS may adopt lower impact behaviors, keeping visitation activities or business affairs more orderly, smoothly, and eco-friendly when the visitor education interventions are highly effective. Hence, the effective visitor educational interventions may enhance the capacity of ETS with the same tourism resources and facilities. The different intensity of visitor education intervention results in different maximum capacity of Ecological Tourism System. $K$ is the maximum capacity of ETS with specific tourism resources and visitor education interventions. Hence, $K$ is stochastically perturbed by the visitor education as follows:

$$
\frac{1}{K} \longrightarrow \frac{1}{K}+\varepsilon_{4} \omega_{4}(t)
$$

where $\omega_{4}(t)$ and $\varepsilon_{4}$ represent a Brownian motion and the intensity of visitor education, respectively.

Then, the Multiparameter Stochastic Dynamics of Ecological Tourism System (MPSDETS) perturbed by visitor educational interventions may be described by Ito equation as follows:

$$
d x=x\left[\left(\alpha+\beta-\gamma-\frac{1}{K} x\right) d t+x^{2} \varepsilon d \omega^{T}(t)\right],
$$

where $\varepsilon=\left(\varepsilon_{1}, \varepsilon_{2}, \varepsilon_{3}, \varepsilon_{4}\right)$ and $\omega(t)=\left(\omega_{1}(t), \omega_{2}(t), \omega_{3}(t), \omega_{4}(t)\right)$.

\section{The Solutions of MPSDETS-Model}

With any specified definition, $\left(\Omega, \mathscr{F},\left\{\mathscr{F}_{t}\right\}_{t \geq 0}, \mathscr{P}\right)$ is denoted as a complete probability space with a filtration $\left\{\mathscr{F}_{t}\right\}_{t \geq 0}$. $\left\{\mathscr{F}_{t}\right\}_{t \geq 0}$ satisfies the right continuous and $\mathscr{F}_{0}$ contains all pnull sets $[29,30] . \omega(t)$ is denoted as a multidimensional standard Brownian motion defined on the proceeding probability space.

Because $x(t)$ is the total number of stakeholders, it should be nonnegative. Furthermore, if the stochastic differential equation has a unique global solution for any given initial value $x_{0}$, the coefficients of the equation should satisfy the linear growth condition and the local Lipschitz condition [31-34]. But the coefficients only satisfy the locally Lipschitz continuous condition, so the solution of (9) may explode at a finite time $[31,34]$.

The purpose of this section is to discover whether visitor education interventions can suppress the explosion of dynamic system or not. It is natural to impose the hypothesis on the visitor education intensities as follows:

$$
\text { (H1) } \varepsilon_{i} \neq 0, \quad(i=1, \ldots, 4) .
$$

The following theorem reveals that visitor education interventions can suppress the explosion of the solution as suggested by Wei et al. (2013). In other words, the theorem shows that the solution of (9) is positive and global under the hypothesis (H1).

Theorem 1. If $\varepsilon_{i} \neq 0,(i=1, \ldots, 4)$, for any system parameters $\alpha, \beta, \gamma, K \in R^{+}$and any given initial value $x_{0} \in R^{+}$, there is a unique value $x(t)$ to (9) on $t \geq 0$ and the solution is positive with probability one; namely, $x(t)>0$ when $t \geq 0$ almost surely.

Proof. Because the coefficients of (9) satisfy the locally Lipschitz continuous, there is a local solution $x(t)$ on $\left[0, \tau_{e}\right)$ for any given initial value $x_{0} \in R^{+}$, when $\tau_{e}$ is the explosion time [29]. In order to prove the solution of (9) is global and 
positive, it should be shown that $\tau_{e}=\infty$ almost surely. The initial value of the equation $x_{0}$ varies within the interval $\left[1 / k_{0}, k_{0}\right]$ for any sufficient large positive value $k_{0}$. The stopping time is denoted as

$$
\tau_{k}=\inf \left\{t \in\left[0, \tau_{e}\right): x(t) \notin\left(\frac{1}{k}, k\right), k \geq k_{0}\right\},
$$

where $\inf \emptyset=\infty$ and $\emptyset$ is denoted as the empty set. It is obvious that $\tau_{k}$ is increasing when $k \rightarrow \infty$. One can see that $\tau_{\infty}=\lim _{k \rightarrow \infty} \tau_{k}$; hence, $\tau_{\infty} \leq \tau_{e}$ a.s.

In order to show that $\tau_{e}=\infty$ a.s. and $x(t)>0$ for any $t \geq 0$, we should prove that $\tau_{\infty}=\infty$ firstly. In other words, the equivalent statement of the theorem shows that $\tau_{\infty}=\infty$ a.s. If the statement $\tau_{\infty}=\infty$ a.s. is not true, then there is a pair of constant $T \geq 0$ and $\varepsilon \in(0,1)$ such that

$$
P\left\{\tau_{\infty} \leq T\right\}>\varepsilon
$$

Hence, there is an integer $k_{1} \geq k_{0}$ such that

$$
P\left\{\tau_{\infty} \leq T\right\}>\varepsilon \quad \forall k \geq k_{1} .
$$

The $C^{2}$-function is defined as $V: R^{+} \rightarrow R^{+}$

$$
V(x)=\sqrt{x}-1-\log x^{0.5}
$$

The function $V(x)$ is nonnegative on $x>0$.

Since $V^{\prime}(x)=1-1 / x=0$ and $\left.V^{\prime \prime}(x)\right|_{x=1}=1>0$, it can be derived from the first or second order condition that $V(x)>0$ for any $x>0$. When $x(t) \in R^{+}$, it can be derived from the Ito formula that

$$
d V(x)=F(x) d t+0.5\left(x^{1.5}-x\right) \varepsilon d \omega^{T}(t),
$$

where

$$
\begin{aligned}
& F(x)=\left[0.5\left(x^{-0.5}-x^{-1}\right) x\left(\alpha+\beta-\gamma-\frac{1}{K} x\right)\right. \\
& \left.+\left(0.25 x^{-2}-0.125 x^{-1.5}\right) x^{4} \varepsilon \varepsilon^{T}\right]=0.5\left(x^{0.5}-1\right) \\
& +\left(\alpha+\beta-\gamma-\frac{1}{K} x\right)+\left(0.25 x^{2}\right. \\
& \left.-0.125 x^{2.5}\right) \varepsilon \varepsilon^{T}=-0.25 \varepsilon \varepsilon^{T} x^{2.5}+0.25 \varepsilon \varepsilon^{T} x^{2}+\frac{1}{k} x \\
& -\frac{1}{2 k} x^{1.5}+0.5(\alpha+\beta-\gamma) x^{0.5}-(\alpha+\beta-\gamma) \\
& \leq-0.25 \varepsilon \varepsilon^{T} x^{2.5}+0.25 \varepsilon \varepsilon^{T} x^{2}+\frac{1}{k} x+\frac{1}{2 k} x^{1.5} \\
& +0.5|(\alpha+\beta-\gamma)| x^{0.5}+|\alpha+\beta-\gamma| .
\end{aligned}
$$

Recalling the hypothesis (H1), it is not difficult to see that $F(x)$ is bounded by sufficient large positive value $M$. Therefore, it can be obtained that

$$
d V(x) \leq M+0.5\left(x^{1.5}-x\right) \varepsilon d \omega^{T}(t) .
$$

Integrating both sides of proceeding inequality from 0 to $T \wedge$ $\tau_{k}$, we can yield

$$
\begin{aligned}
\int_{0}^{T \wedge \tau_{k}} d V(x) \leq & \int_{0}^{T \wedge \tau_{k}} M d t \\
& +\int_{0}^{T \wedge \tau_{k}} 0.5\left(x^{1.5}-x\right) \varepsilon d \omega^{T}(t) .
\end{aligned}
$$

And then take expectations on the both sides of inequality (18), and we can yield

$$
\begin{aligned}
E V\left(x\left(T \wedge \tau_{k}\right)\right) & \leq v\left(x_{0}\right)+M E\left(T \wedge \tau_{k}\right) \\
& \leq v\left(x_{0}\right)+M T,
\end{aligned}
$$

$\Omega_{k}=\left\{\tau_{k} \leq T\right\}$ for $k \geq k$ and $P\left(\tau_{\infty} \leq T\right)>\varepsilon$, so that $P\left(\Omega_{k}\right)>\varepsilon$. Take any $\xi \in \Omega_{k}$, by the definition of stopping time (11), and there is $x\left(\tau_{k}, \xi\right)$ which is equal to either $1 / k$ or $k$. Then, we can derive that $V\left(x\left(\tau_{k}, \xi\right)\right)$ is not less than either

$$
\begin{gathered}
\sqrt{k}-1-0.5 \log k \\
\text { or } \sqrt{\frac{1}{k}}-1-0.5 \log \left(\frac{1}{k}\right) .
\end{gathered}
$$

So, there is $x(t) \geq k$ or $x(t) \leq 1 / k$ when $t \geq \tau_{k}$. Thus, we can get

$$
\begin{aligned}
V\left(x\left(\tau_{k}, \xi\right)\right) \geq & {[\sqrt{k}-1-0.5 \log k] \wedge \sqrt{\frac{1}{k}}-1 } \\
& -0.5 \log \left(\frac{1}{k}\right) .
\end{aligned}
$$

Taking expectation on both sides of (20), we can yield

$$
\begin{aligned}
E V\left(x\left(\tau_{k}, \xi\right)\right) \geq & {[\sqrt{k}-1-0.5 \log k] \wedge \sqrt{\frac{1}{k}}-1 } \\
& -0.5 \log \left(\frac{1}{k}\right) .
\end{aligned}
$$

Then, we can get the following inequality from (22):

$$
\begin{aligned}
v\left(x_{0}\right)+M T \geq & E V\left(x\left(T \wedge \tau_{k}\right)\right) \\
\geq & {[\sqrt{k}-1-0.5 \log k] \wedge \sqrt{\frac{1}{k}}-1 } \\
& -0.5 \log \left(\frac{1}{k}\right) .
\end{aligned}
$$

Moreover, $T$ is a constant which is denoted in (12), so that $v\left(x_{0}\right)+M T<\infty$. When $k \rightarrow \infty$, we can obtain

$$
\begin{aligned}
\infty> & v\left(x_{0}\right)+M T \\
\geq & \lim _{k \rightarrow \infty}[\sqrt{k}-1-0.5 \log k] \wedge \sqrt{\frac{1}{k}}-1 \\
& \quad-0.5 \log \left(\frac{1}{k}\right)=\infty .
\end{aligned}
$$


Then, we can get the contradiction by (24). So, we can draw a conclusion that the statement of (12) is false. Furthermore, the original statement is true which is $P\left\{\tau_{k} \leq \tau_{\infty}\right\}<\varepsilon$. In other words, the statement $\tau_{\infty}=\infty$ is true almost surely.

It is easy to derive from Theorem 1 that the solution of (9) exists and nerve explodes in a finite time. Following hypothesis (11), the visitor educational interventions on ETS can introduce stochastic parameters including stochastic intrinsic increasing rate of stakeholders and stochastic emigration rate of stakeholders, stochastic immigration rate of stakeholders, and stochastic maximum capacity of tourism ecological system. When $\varepsilon_{i} \neq 0,(i=1, \ldots, 4)$, the stochastic parameters can suppress the explosion of the solution. It means that the slight or tiny stochastic change of any parameter introduced by the visitor educational intervention can maintain the stability and sustainability of the ETS. Therefore, the visitor educational intervention is necessary condition of safe and stable developing of ETS. Without the visitor education intervention, the system would collapse in a finite time as mentioned in Section 1. For instance, tens of thousands of tourists were trapped in the Huashan Mountain because of the conflict between visitors and park mangers.

\section{The Bounded Property of MPSDETS-Model}

Under the hypothesis (H1), Theorem 1 shows that the solution of MPSDETS (9) does not explode in a finite time. The next step is to show that the solution varies in a finite interval. $x\left(t, x_{0}\right)$ is denoted as the unique global solution of (9) for any given initial value $x_{0}>0$. The stochastically ultimate bounded property of the solution of the MPSDETS is defined as follows.

Definition 2. For any initial value $x_{0}>0$, if the solution of (9) $x\left(t, x_{0}\right)$ has the property that

$$
\lim _{t \rightarrow \infty} \inf x\left(t, x_{0}\right)>0,
$$

then (9) is stochastically and ultimately bounded with probability one. It is useful to present the following theorem which shows that (9) is stochastically ultimate bounded.

Theorem 3. If the coefficients of (9) satisfy the property as follows:

$$
\begin{aligned}
K & <\frac{1}{\varepsilon \varepsilon^{T}}, \\
(\alpha+\beta-\gamma)^{2}+\left(4 \varepsilon \varepsilon^{T}-\frac{6}{K}\right)+\frac{4}{K^{2}} & \leq 0,
\end{aligned}
$$

then the solution of (9) $x\left(t, x_{0}\right)$ is stochastically ultimate bounded with probability one. Meanwhile, given any initial value $x_{0} \in R^{+}$, the solution of (9) has the property that

$$
\lim _{t \rightarrow \infty} \sup x\left(t, x_{0}\right)<\infty .
$$

Proof. For convenience, we can define $C^{2}$-function $V: R^{+} \rightarrow$ $R^{+}$by

$$
V(x)=x-1-\log x .
$$

By Ito formula, we can get

$$
\begin{gathered}
d V(x)=\left(\left(\varepsilon \varepsilon^{T}-\frac{1}{K}\right) x^{2}+\left(\alpha+\beta-\gamma+\frac{1}{K}\right) x\right. \\
-(\alpha+\beta-\gamma)) d t+\left(x^{2}-x\right) \varepsilon d d \omega^{T}(t) .
\end{gathered}
$$

$L V(x(t))$ is denoted as

$$
\begin{aligned}
L V(x(t))= & \left(\varepsilon \varepsilon^{T}-\frac{1}{K}\right) x^{2}+\left(\alpha+\beta-\gamma+\frac{1}{K}\right) x \\
& -(\alpha+\beta-\gamma) .
\end{aligned}
$$

Obviously, $L V(x(t))$ is quadratic function. Applying the properties of quadratic function, the following inequalities can be obtained:

$$
\begin{aligned}
\varepsilon \varepsilon^{T}-\frac{1}{K}< & 0, \\
\Delta= & \left(\alpha+\beta-\gamma-\frac{1}{K}\right)^{2} \\
& +4\left(\varepsilon \varepsilon^{T}-\frac{1}{K}\right)(\alpha+\beta-\gamma) \leq 0 \\
= & (\alpha+\beta-\gamma)^{2}+\left(4 \varepsilon \varepsilon^{T}-\frac{6}{K}\right)+\frac{4}{K^{2}} \leq 0 .
\end{aligned}
$$

So, when (26) are satisfied, the quadratic function $L V(x(t))$ must be less than zero. Therefore, we can obtain that

$$
d V(x(t)) \leq 0 d t+\left(x^{2}-x\right) \varepsilon d d \omega^{T}(t) .
$$

Integrating both sides of inequality (33) from 0 to $t$, we can yield

$$
\begin{array}{r}
\int_{0}^{t} d V(x(t)) \leq \int_{0}^{t} 0 d t+\int_{0}^{t}\left(x^{2}-x\right) \varepsilon d d \omega^{T}(t), \\
V(x(t)) \leq V\left(x_{0}\right)+\int_{0}^{t}\left(x^{2}-x\right) \varepsilon d d \omega^{T}(t) .
\end{array}
$$

$x(t)$ is defined as

$$
x(t) \triangleq V\left(x_{0}\right)+\int_{0}^{t}\left(x^{2}-x\right) \varepsilon d d \omega^{T}(t) .
$$

Applying Doob's martingale convergence theorem, we can yield

$$
\lim _{t \rightarrow \infty} x(t)<\infty \quad \text { a.s. }
$$

Then, $\lim _{t \rightarrow \infty} \sup x(t)<\infty$ a.s. is obtained.

Recalling the definition of $V(x)=x-1-\log x$, if and only if $x \rightarrow \infty$ or $x \rightarrow 0$, the limit of $V(x)$ is $\infty$. Hence, $\lim _{t \rightarrow \infty} \sup V(x(t))=\lim _{t \rightarrow \infty} \sup (x(t)-1-\log (x(t)))<\infty$ a.s.

Then, it is not difficult to see that

$$
0<\lim _{t \rightarrow \infty} \inf x(t)<\lim _{t \rightarrow \infty} \sup x(t)<\infty \quad \text { a.s. }
$$




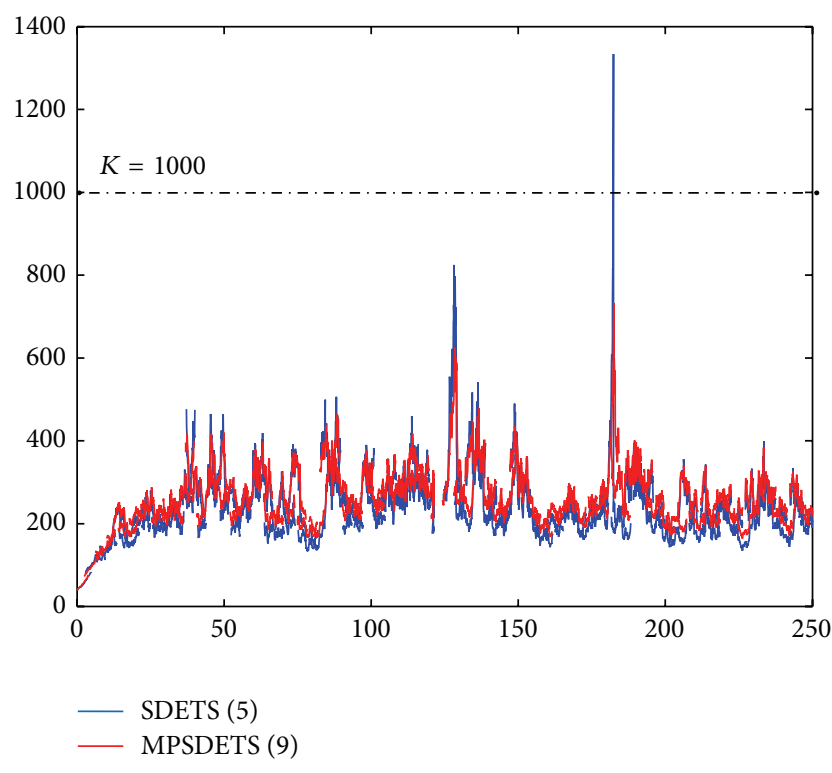

FIgURE 3: The simulations of SDETS (5) and MPSDETS (9) when $\alpha=0.25, \beta=0.09, \gamma=0.06, K=1000$, and $x_{0}=30$.

Given any initial value $x_{0}$, it is easy to obtain that the solution of (9) $x(t)$ varies in the interval of $\left[\lim _{t \rightarrow \infty}\right.$ inf $x(t)$, $\left.\lim _{t \rightarrow \infty} \sup x(t)\right]$ as shown in Theorem 3. So, the stakeholders can develop within an interval steadily. The population of stakeholders can be retained in a safe interval. ETS under visitor educational interventions can keep sustainable opportunities for high quality visitor experiences while avoiding or minimizing associated negative impacts to protected area resources although there are very large tourists in a short time. In other words, the visitation negative impacts in protected areas caused by large amount of visitors in public vocations can be minimized by the high efficient visitor education programs.

\section{Simulations}

Following the Euler-Maruyama method [35], it is easy to get the numerical solutions of MPSDTES (9) with different parameters by MATLAB program. And the figures can be drawn according to the simulation data.

Figure 3 shows that SDETS (2) can effectively suppress the explosion of Ordinary Differential Equation (1) when $\alpha=0.25, \beta=0.09, \gamma=0.06, K=1000$, and $x_{0}=30$. But SDETS (2) cannot avoid some chaos situations either. For example, the peak value of visitors and other stakeholders $x_{\max }(184)=1500$ is larger than the maximum capacity $K=$ 1000 . Although $x(t)$ decreases to $x(185)=780$, the system is chaotic in the short interval time $t=[183,185]$. The chaos situations appear two times in an interval time $t \in[0,250]$ when $\alpha=0.25, \beta=0.09, \gamma=0.06, K=1000$, and $x_{0}=30$. Tables $1(\mathrm{a})$ and $1(\mathrm{~b})$ show that chaos situations are getting larger when given larger initial value $x_{0}$. The times of chaos situations are smaller when given smaller $\alpha$ comparing Tables $1(\mathrm{a})$ and $1(\mathrm{~b})$.
TABLE 1: The over maximum capacity of Ecological Tourism System times $(x(t)>1000)$.

(a)

\begin{tabular}{lccccc}
\hline & \multicolumn{5}{c}{$\alpha=0.25, \beta=0.09, \gamma=0.06, K=1000$} \\
\hline$x_{0}$ & 30 & 500 & 800 & 900 & 950 \\
\hline SDETS (2) & 2 & 2 & 3 & 5 & 7 \\
\hline MPSDTES (9) & 0 & 0 & 0 & 0 & 0 \\
\hline
\end{tabular}

(b)

\begin{tabular}{lccccc}
\hline & \multicolumn{6}{c}{$\alpha=0.15, \beta=0.07, \gamma=0.06, K=1000$} \\
\hline$x_{0}$ & 30 & 500 & 900 & 950 & 999 \\
\hline SDETS (2) & 0 & 0 & 1 & 2 & 2 \\
\hline MPSDTES (9) & 0 & 0 & 0 & 0 & 0 \\
\hline
\end{tabular}

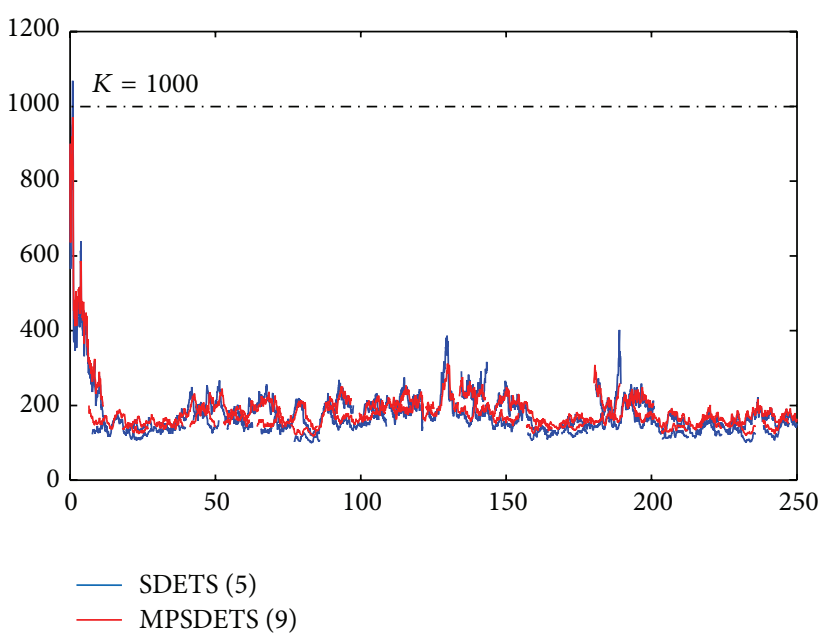

FIGURE 4: The simulations of SDETS (5) and MPSDETS (9) when $\alpha=0.15, \beta=0.07, \gamma=0.04, K=1000$, and $x_{0}=900$.

Figure 3 shows that MPSDETS (9) can also effectively suppress the explosion of ODETS (1) when $\alpha=0.25, \beta=$ $0.09, \gamma=0.06, K=1000$, and $x_{0}=30$. MPSDETS (9) can keep the peak value of visitors and other stakeholders under the maximum capacity of Ecological Tourism System compared with SDETS (2). The chaos situations disappear in the interval $t \in[0,250]$ with the same parameters. Table $1(\mathrm{a})$ shows that MPSDETS (9) can reduce the times of chaos situation obviously. For example, SDETS (2) has 5 times of chaos situation when $x_{0}=900$, but MPSDETS (9) has none. Figure 4 also shows the same results with $\alpha=0.15, \beta=0.07$, $\gamma=0.04, K=1000$, and $x_{0}=900$.

It is interesting to find that MPSDETS (9) is much more stable than SDETS (2) from the simulations. The simulations also show that ETS can develop more sustainably when all parameters are perturbed by continuous visitor educational intervention. So, the stakeholders in ETS should be involved in the visitor education programs. The stakeholders such as 
the visitation product sellers group and the special interest group play necessary roles in visitor education programs.

\section{Conclusions}

Section 1 concludes that the fast expanding visitation in protected areas causes some serious natural resource and culture impacts in China and other countries. The popular management strategies to deal with the negative impacts include regulations, site managements, and visitor education programs. The regulation and site management gain very limit efficacy. It is easy to conclude from literatures that the fast expanding visitation in parks and the protected area including some natural history park is quickly damaged without visitor education. And the visitor education intervention programs are the most effective way to deal with negative visitation impacts as shown in the literatures. Researchers only discussed visitor educational message content, delivery, and audience characteristics, and so on. However, there are few mathematical models to study the efficacy of visitor education interventions in views of dynamics of ETS. So, it is valuable to study the dynamics of ETS with one stochastic parameter under visitor education interventions. A similar study has been done by Wei et al. in 2013 .

In Section 2, ODETS is established to describe Ecological Tourism System without visitor educational intervention. The model reveals that the ecological tourism collapses without any perturbed factors such as visitor educational intervention. SDETS is effective to suppress the explosion of ODETS as shown in Figures 1 and 2. But it is not effective to suppress the peak value of visitors as shown in Figure 3. The peak value of visitors is larger than the maximum capacity of the tourism system in a short time. Hence, it is necessary to assume that the intrinsic increasing rate of stakeholders, the immigration rate of stakeholders from outside of the system, and the emigration rate of stakeholders from the inner system are stochastically perturbed by the visitor education interventions. Then, the Multiparameter Stochastic Dynamics of ETS perturbed by visitor educational interventions can be described by Ito equation.

In Sections 3 and 4, we prove that MPSDETS does not explode in a finite time. Then, we prove that the solution of MPSDETS varies in a finite interval. The solution shows that the population of the system retains in an acceptable level which can sustain the opportunities for high quality visitor experiences while avoiding or minimizing the associated negative impacts to the protected area resources and neighbors communities. The educational interventions increase visitors' knowledge and alter visitors' undesired behaviors to low impact behaviors. In other words, the tourism system can develop steadily and safely even under a large amount of visitors in public vocations when employing visitor education intervention programs.

\section{Conflict of Interests}

The authors declare that there is no conflict of interests regarding the publication of this paper.

\section{Acknowledgments}

This work is supported by the National Science Fund of China (Grant no. 71272177/G020902, Grant no. 31100958), Social Science Foundation of Guangdong (Grant no. GD14XGL03), Youth Innovation Fund of Shenzhen Polytechnic (Grant no. 601422K35018), Project of Higher Vocational Education Research Society of China (Grant no. GZYLX1213345), and Philosophical and Social Science Project of Shenzhen (Grant no. 125C09). The authors would like to thank the editor and an anonymous referee for their helpful comments on the paper.

\section{References}

[1] G. L. Peterson and D. W. Lime, "People and their behavior: a challenge for recreation management," Journal of Forestry, vol. 77, no. 6, pp. 343-347, 1979.

[2] J. E. Frost and S. F. McCool, "Can visitor regulations enhance recreational experiences?” Environmental Management, vol. 12, no. 1, pp. 5-9, 1988.

[3] R. Buckley, "Evaluating the net effects of ecotourism on the environment: a framework, first assessment and future research," Journal of Sustainable Tourism, vol. 17, no. 6, pp. 643672, 2009.

[4] C. Wyborn, "Managing change or changing management: climate change and human use in Kosciuszko National Park," Australasian Journal of Environmental Management, vol. 16, no. 4, pp. 208-217, 2009.

[5] D. D. White, R. J. Virden, and C. J. van Riper, "Effects of place identity, place dependence, and experience-use history on perceptions of recreation impacts in a natural setting," Environmental Management, vol. 42, no. 4, pp. 647-657, 2008.

[6] R. Buckley, "Sustainable tourism: research and reality," Annals of Tourism Research, vol. 39, no. 2, pp. 528-546, 2012.

[7] W. M. Vagias, R. B. Powell, D. D. Moore, and B. A. Wright, "Predicting behavioral intentions to comply with recommended leave no trace practices," Leisure Sciences, vol. 36, no. 5, pp. 439457, 2014.

[8] T. J. Brown, S. H. Ham, and M. Hughes, "Picking up litter: an application of theory-based communication to influence tourist behaviour in protected areas," Journal of Sustainable Tourism, vol. 18, no. 7, pp. 879-900, 2010.

[9] J. L. Marion and S. E. Reid, "Minimising visitor impacts to protected areas: the efficacy of low impact education programmes," Journal of Sustainable Tourism, vol. 15, no. 1, pp. 5-27, 2007.

[10] D. Waayers, D. Newsome, and D. Lee, "Observations of noncompliance behaviour by tourists to a voluntary code of conduct: a pilot study of turtle tourism in the Exmouth region, Western Australia," Journal of Ecotourism, vol. 5, no. 3, pp. 211222, 2006.

[11] S.-W. Wen and Z.-W. Wu, "Effects of ecological educational on recreational impacts," Acta Ecologica Sinica, vol. 29, no. 2, pp. 768-775, 2009 (Chinese).

[12] A. W. Whitworth, "An investigation into the determining factors of zoo visitor attendances in UK zoos," PLoS ONE, vol. 7, no. 1, Article ID e29839, p. 230, 2012.

[13] D. Hewlett and J. Edwards, "Beyond prescription: community engagement in the planning and management of National Parks as tourist destinations," Tourism Planning \& Development, vol. 10, no. 1, pp. 45-63, 2013. 
[14] S. F. McCool and D. W. Lime, "Tourism carrying capacity: tempting fantasy or useful reality?" Journal of Sustainable Tourism, vol. 9, no. 5, pp. 372-388, 2001.

[15] C. A. Monz, D. N. Cole, Y.-F. Leung, and J. L. Marion, "Sustaining visitor use in protected areas: future opportunities in recreation ecology research based on the USA experience," Environmental Management, vol. 45, no. 3, pp. 551-562, 2010.

[16] S. Qiang, L. Chong-gui, and D. Jin-yang, "Assessment of impacts of visitors' activities on vegetation in Zhangjiajie National Forest Park," Journal of Forestry Research, vol. 13, no. 2, pp. 137-140, 2002.

[17] S. Spanou, K. Tsegenidi, and T. Georgiadis, "Perception of visitors' environmental impacts of ecotourism: a case study in the Valley of Butterflies protected area, Rhodes Island, Greece," International Journal of Environmental Research, vol. 6, no. 1, pp. 245-258, 2012.

[18] J. W. Roggenbuck and D. L. Berrier, "Communications to disperse wilderness campers," Journal of Forestry, vol. 79, no. 5, pp. 295-297, 1981.

[19] H. Mannan, C. Boostrom, M. MacLachlan, E. McAuliffe, C. Khasnabis, and N. Gupta, "A systematic review of the effectiveness of alternative cadres in community based rehabilitation," Human Resources for Health, vol. 10, article 20, 2012.

[20] D. M. Kuehn, P. D. D'Luhosch, V. A. Luzadis, R. W. Malmsheimer, and R. M. Schuster, "Attitudes and intentions of off-highway vehicle riders toward trail use: implications for forest managers," Journal of Forestry, vol. 109, no. 5, pp. 281-287, 2011.

[21] M. S. Bank, J. R. Burgess, D. C. Evers, and C. S. Loftin, "Mercury contamination of biota from Acadia National Park, Maine: a review," Environmental Monitoring and Assessment, vol. 126, no. 1-3, pp. 105-115, 2007.

[22] M. Ruchter, B. Klar, and W. Geiger, "Comparing the effects of mobile computers and traditional approaches in environmental education," Computers and Education, vol. 54, no. 4, pp. 10541067, 2010.

[23] W. Powell, M. Vagias, D. Moore, and B. Wright, "Development, psychometric qualities, and cross-validation of the leave no trace attitudinal inventory and measure," Journal of Leisure Research, vol. 44, no. 2, pp. 234-256, 2012.

[24] K. T. Hegetschweiler, N. van Loon, A. Ryser, H.-P. Rusterholz, and B. Baur, "Effects of fireplace use on forest vegetation and amount of woody debris in suburban forests in Northwestern Switzerland," Environmental Management, vol. 43, no. 2, pp. 299-310, 2009.

[25] D. N. Cole, T. P. Hammond, and S. F. McCool, "Information quantity and communication effectiveness: low-impact messages on wilderness trailside bulletin boards," Leisure Sciences, vol. 19, no. 1, pp. 59-72, 1997.

[26] J. Curtis, S. H. Ham, and B. Weiler, "Identifying beliefs underlying visitor behaviour: a comparative elicitation study based on the theory of planned behaviour," Annals of Leisure Research, vol. 13, no. 4, pp. 564-589, 2010.

[27] A. T. McDonald, P. J. Chapman, and K. Fukasawa, "The microbial status of natural waters in a protected wilderness area," Journal of Environmental Management, vol. 87, no. 4, pp. 600-608, 2008.

[28] B. Bednar-Friedl, D. A. Behrens, and M. Getzner, "Optimal dynamic control of Visitors and endangered species in a national park," Environmental \& Resource Economics, vol. 52, no. 1, pp. 1-22, 2012.
[29] D. Wei, S. Wen, Y. Chen, Y. Xu, and B. Liang, "The stochastic dynamics for ecological tourism system with visitor educational intervention," Mathematical Problems in Engineering, vol. 2013, Article ID 617608, 9 pages, 2013.

[30] X. Mao, Stochastic Differential Equations and Applications, Harwood Publishing, Chichester, UK, 2007.

[31] R. S. Liptser and A. N. Shiryayev, Theory of Martingales, Springer Netherlands, Rotterdam, The Netherlands, 1989.

[32] X. Mao, "Almost sure exponential stability for delay stochastic differential equations with respect to semimartingales," Stochastic Analysis \& Applications, vol. 9, no. 2, pp. 177-194, 2007.

[33] X. M. A. Shah, "Exponential stability of stochastic differential delay equations," Stochastics \& Stochastic Reports, vol. 60, no. 1, pp. 135-153, 2007.

[34] C. Jarzynski, "Nonequilibrium work relations: foundations and applications," The European Physical Journal B: Condensed Matter and Complex Systems, vol. 64, no. 3-4, pp. 331-340, 2008.

[35] P. Kloeden and E. Platen, Numerical Solution of Stochastic Differential Equations, Springer, Berlin, Germany, 1999. 


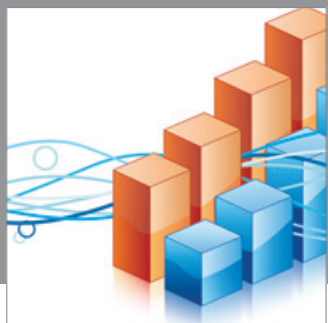

Advances in

Operations Research

mansans

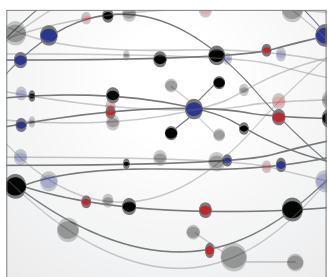

The Scientific World Journal
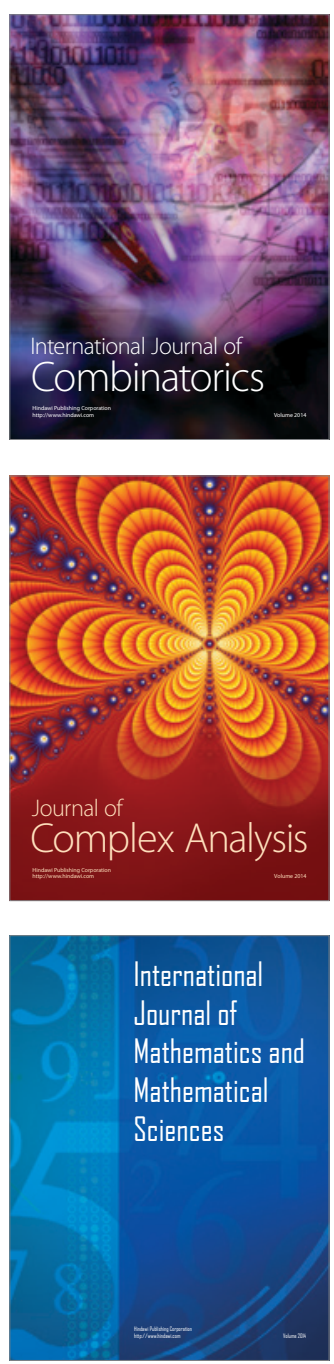
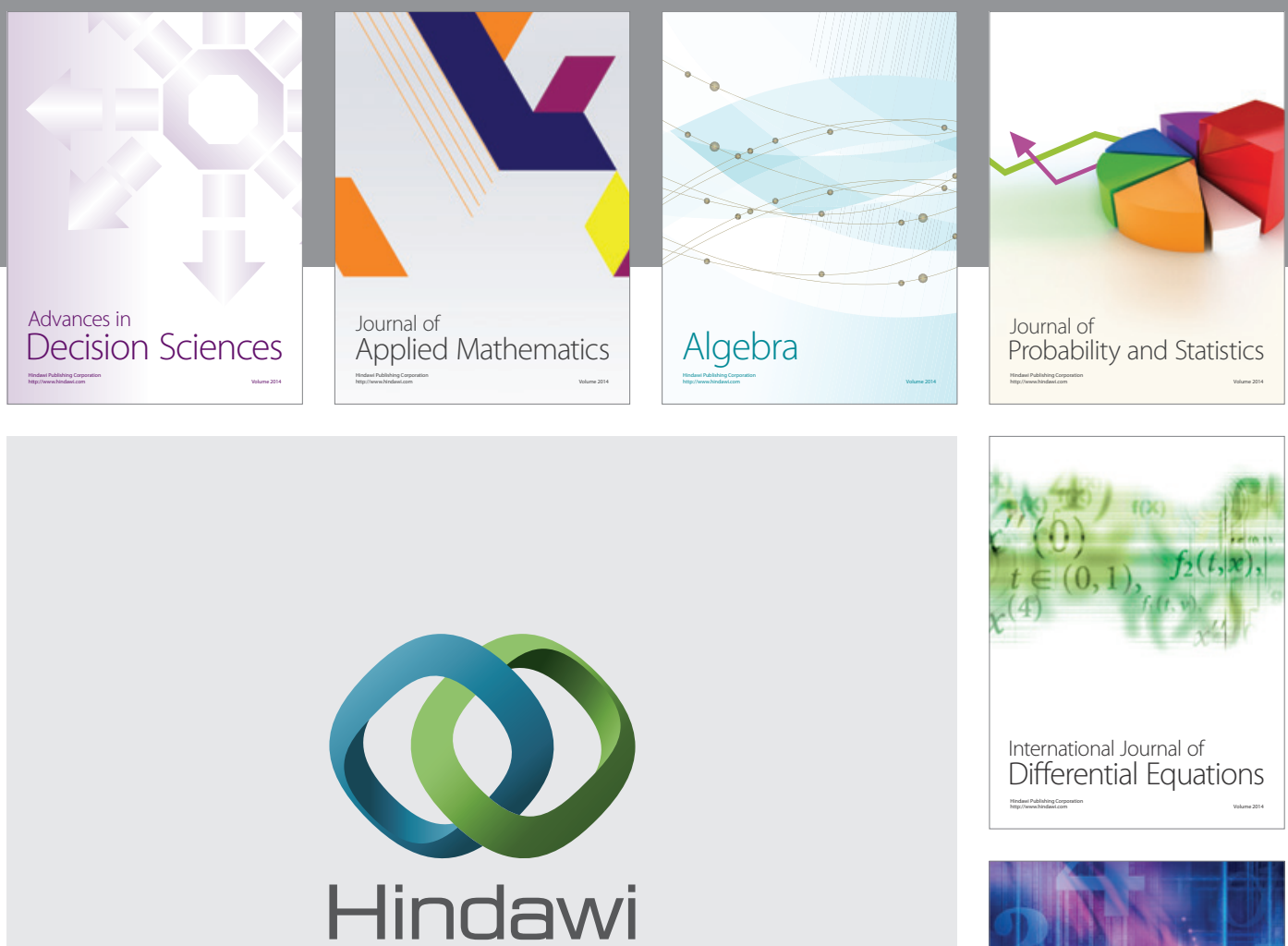

Submit your manuscripts at http://www.hindawi.com
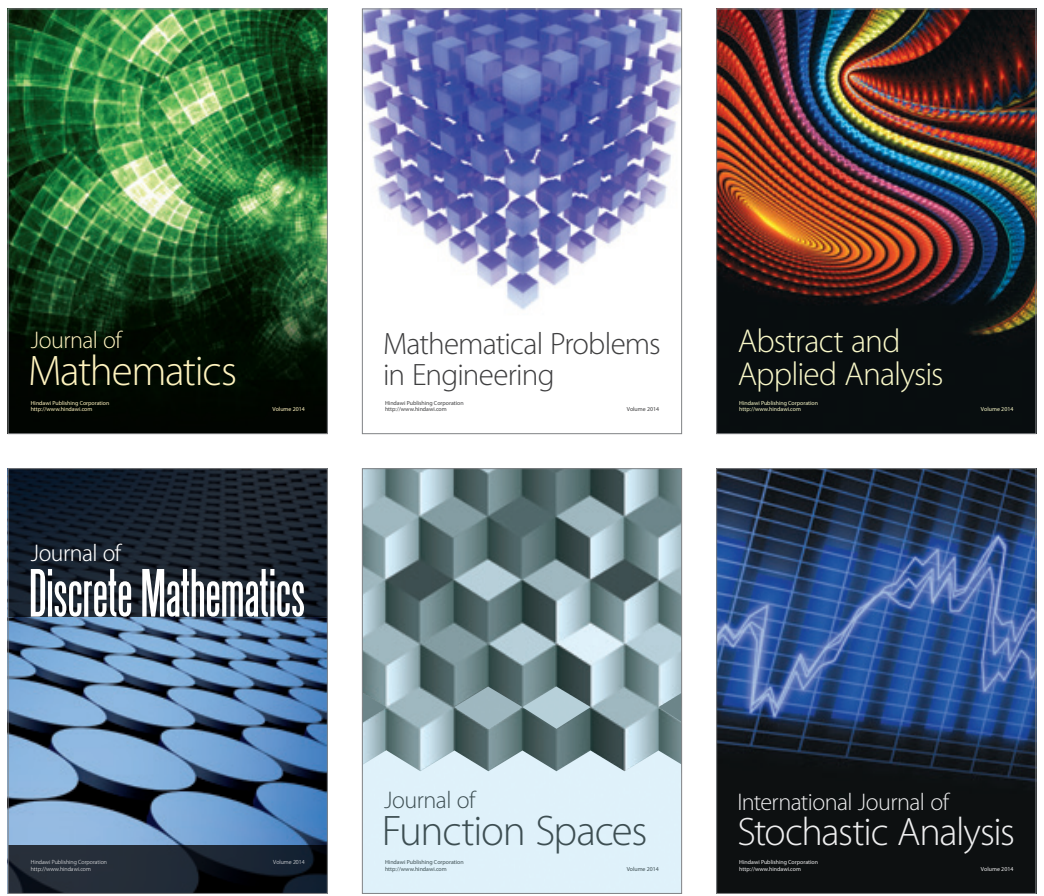

Journal of

Function Spaces

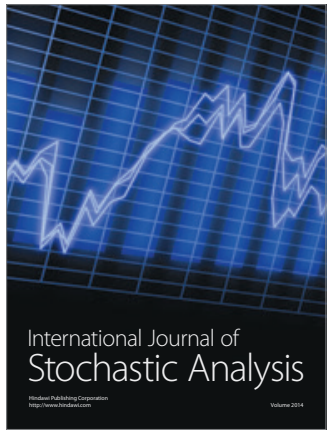

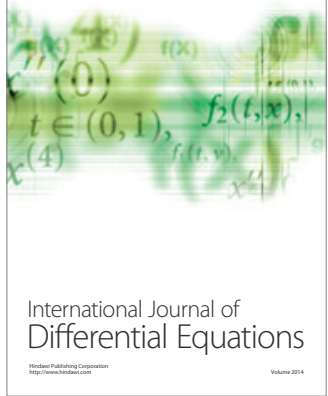
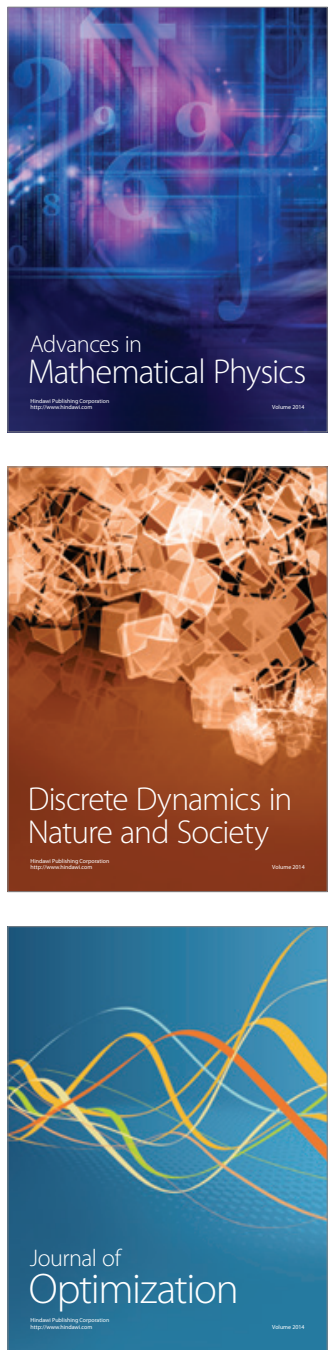